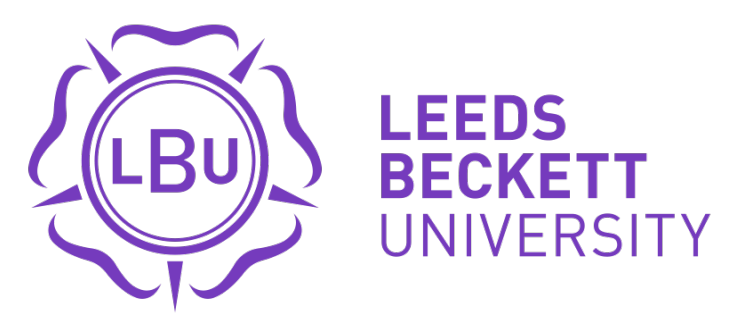

Citation:

Golonka, S and Wilson, AD (2019) Ecological mechanisms in cognitive science. Theory and Psychology, 29 (5). pp. 676-696. ISSN 0959-3543 DOI: https://doi.org/10.1177/0959354319877686

Link to Leeds Beckett Repository record:

https://eprints.leedsbeckett.ac.uk/id/eprint/6545/

Document Version:

Article (Accepted Version)

The aim of the Leeds Beckett Repository is to provide open access to our research, as required by funder policies and permitted by publishers and copyright law.

The Leeds Beckett repository holds a wide range of publications, each of which has been checked for copyright and the relevant embargo period has been applied by the Research Services team.

We operate on a standard take-down policy. If you are the author or publisher of an output and you would like it removed from the repository, please contact us and we will investigate on a case-by-case basis.

Each thesis in the repository has been cleared where necessary by the author for third party copyright. If you would like a thesis to be removed from the repository or believe there is an issue with copyright, please contact us on openaccess@leedsbeckett.ac.uk and we will investigate on a case-by-case basis. 


\section{Ecological Mechanisms in Cognitive Science}

Sabrina Golonka*

\&

Andrew D Wilson

*Corresponding author details:

Leeds School of Social Sciences, Leeds Beckett University, Leeds UK

Email: s.golonka@leedsbeckett.ac.uk

Web: https://cognitioninaction.wordpress.com/

Twitter: @PsychScientists 


\begin{abstract}
Bechtel \& Abrahamsen (2010) defined and described what it means to be a dynamic causal mechanistic explanatory model. They discussed the development of a mechanistic explanation of circadian rhythms as an exemplar of the process, and they challenged cognitive science to follow this example. This paper takes on that challenge.

A mechanistic model is one that accurately represents the real parts and operations of the mechanism being studied. These real components must be identified by an empirical programme that decomposes the system at the correct scale and localises the components in space and time. Psychological behaviour emerges from the nature of our real time interaction with our environments, and so here we show that the correct scale to guide decomposition is picked out by the ecological perceptual information that enables that interaction. As proof of concept, we show that a simple model of coordinated rhythmic movement, grounded in information, is a genuine dynamical mechanistic explanation of many key coordination phenomena.
\end{abstract}




\section{Introduction}

Not all explanations are equal. Cognitive models are primarily functional, 'how-possibly' models of system capacities. These models represent one way a system that exhibits a target capacity might function, and they can be useful first steps, but they may not correspond in any meaningful way to the causal structure underpinning the system of interest.

The gold standard 'how-actually' explanation for science is called a dynamic causal mechanistic explanation (e.g. Bechtel \& Abrahamson, 2010). These are scientific theories, usually implemented in a quantitative model, in which each part and operation of the model explicitly stands in for real parts and operations (collectively, real components) of the system to be explained. When they are an option, causal mechanistic models offer a number of explanatory advantages that a scientific discipline can benefit greatly from (Bechtel and Abrahamson, 2010);

1) demonstrate that a given mechanism is sufficient to produce the target phenomenon

2) explore the functioning of the mechanism in a larger parameter space than is accessible in experiments

3) identify whether candidate parts are essential to the mechanism's functioning

4) explore how particular types of damage might affect the system by perturbing the model in particular ways

5) to explain how coordinated behavior can emerge from the coupling of simpler mechanisms

6) to explore the consequences of altering the relations between multiple mechanisms

Compared to other explanation types (e.g., functional, covering law) only dynamical causal mechanistic models have access to all benefits, most notably the last two. For this reason, if the subject matter is amenable to this type of explanation, it is worth steering research in the direction of dynamic causal mechanistic models. 
In this paper we present a theoretical and methodological framework that can support the development of causal mechanistic explanations of cognition and behaviour. This framework is firmly rooted in James J Gibson's ecological approach to perception and action (Gibson, 1979), with the resulting mechanistic models grounded at the scale of Gibsonian perceptual information. Our analysis has emerged from our work pursuing the implications of the ecological ontology for cognitive science in general (Charles et al, 2014; Golonka, 2015; Golonka \& Wilson, 2012, 2018; Wilson \& Golonka, 2013, 2015; Wilson, 2012, 2014, 2018a, b). It became clear to us that the most appropriate framing to identify what makes the ecological approach distinct was in terms of the types of explanations it made possible. It turns out that ecological explanations are built from parts and processes with an identifiable material basis. This means that the ecological approach is, at least in principle, capable of supporting causal mechanistic explanations.

We will briefly review the characteristics of mechanistic explanations, and then contrast these with characteristics of typical cognitive psychological models. We will then review the characteristics of ecological explanations and evaluate how well they support the development of mechanistic explanations, with reference to a specific worked example (coordinated rhythmic movement). We conclude that the ecological approach is actually very well suited to support mechanistic explanations of cognition and behaviour, and should serve as the framework for future work in this area.

\section{Mechanisms}

Bechtel \& Abrahamsen (2010) define a mechanism like this:

A [dynamic] mechanism is a structure performing a function in virtue of its component parts, component operations, and their organization. The orchestrated functioning of the mechanism, manifested in patterns of change over time in properties of its parts and operations, is responsible for one or more phenomena" 
In order to be a causal mechanistic explanation of this type, a model must accurately represent the actual parts and operations (the real components) of the structure, rather than simply characterise the functions of those components. Parts "typically have locations, sizes, structures, and orientations. They are the kinds of things that have masses, carry charges, and transmit momentum." (Craver, 2007, p. 5). Parts should also have some kind of independent existence outside of the mechanism (Glennan, 1996). If the system of interest was the functioning of a car, causal mechanistic models would model parts like the engine and brake line, while functional models would characterise the capacity to accelerate or the capacity to stop. Operations, in contrast, "are the causal components in mechanisms... [which] can potentially be exploited for the purposes of manipulation and control" (Craver, 2007, p. 6). This definition clarifies why the distinction between part types above is important. Causal components can only be identified among parts that are locatable in space /time, that exist independent of the mechanism. Causal components cannot be identified among functional parts, though filler terms may be used instead.

The research strategy for developing mechanistic models is to empirically identify the real parts (to contrast them from functional parts as described above) and processes of the mechanism. This is a programme of decomposition and localisation (Bechtel \& Richardson, 2010) ${ }^{1}$. Decomposition is breaking the mechanism down into the right parts (carving it up at the joints, as it were). Localisation is figuring out where and when these parts do their work in the mechanism. Complete decomposition and localisation may not always be possible, but they should still be pursued. This process is heuristic; there are no rules for doing it other than 'work hard to check you are breaking things up in the right place'. For example, I can decompose a clock by either systematically unscrewing it into smaller and smaller parts that still do things, or by hitting it with a hammer. While

\footnotetext{
${ }^{1}$ Mechanistic models can exist at various stages of development (sketches, schemata, complete mechanisms; Craver, 2007). It takes time to develop the relevant evidence base and it is often appropriate to model the components you know about as you go.
} 
fun, the latter approach does not leave me with parts that feature in the mechanism of a working clock, and I learn this when I try and fail to get the pieces to exhibit meaningful behaviour.

Bechtel (2008a) offers an interesting analogy to 19th century fermentation research to illustrate this point:

By describing the potential intermediates formed in the process of fermentation as themselves undergoing fermentations, physiologists looked too high. They provided little explanatory gain, since researchers were appealing to the phenomenon to be explained to describe the operations that were to provide the explanation. In contrast, by focusing on the elemental composition of sugar and alcohol and appealing to operations of adding or deleting atoms to explain organic processes such as fermentation, chemists focused too low. The chemists clearly appealed to operations on components in a mechanism to explain the phenomenon, but this approach was under constrained.

p 989.

Researchers eventually identified another way in which to carve up the parts to explain fermentation: biochemistry. Operations relevant to fermentation occur between biochemical entities. Thus, to explain fermentation, researchers needed to find biochemical parts. In this example, the success of the research programme depended upon first identifying the appropriate scale for analysis (biochemistry) and then identifying relevant parts and operations. We suggest below that a similar problem is faced by psychology today, and that the ecological approach offers a solution.

\section{Carving Psychology at the Joints}

The key issue for psychology is not the complexity of the subject - other life sciences such as biology and genetics routinely develop causal mechanistic explanations. Instead, the issue is identifying real 
cognitive components that are identifiable with material transformations. Bechtel has attempted this analysis (e.g. Bechtel, 2008b) but still notes that "mental mechanisms are often most usefully described in terms of the content they carry, not their intrinsic physical features" (pg 48). Weiskopf argues against mechanistic modelling in psychology, noting that "for psychological phenomena... often enough, we have no well-demarcated physical system to decompose, and little idea of the proper parts and operations to use in such a decomposition." (pg 322). Some are even less generous about the scale of the problem. For example, Keijzer (2006) argues that "[c]ognition thus conceived is a useful and pragmatic way of demarcating the cognitive domain. Nevertheless, it comes at great theoretical costs. Most notably...it obstructs a clear linkage between the cognitive domain and particular kinds of material systems" (pg 1593). There is, as yet, no clear cut way to decompose cognitive systems into real parts and operations ${ }^{2}$.

In recognition of this, another variety of psychological model has gained popularity in recent years. Chemero and colleagues have argued extensively that cognitive systems cannot actually be meaningfully decomposed, and that dynamical systems theory (DST) explanations work as an alternative to mechanistic explanations in cognitive science (Chemero \& Silberstein, 2008; Silberstein \& Chemero, 2013; Stepp et al, 2005). For instance, DST models of networks in the brain can successfully capture invariant features of the system while making no reference to any specific parts or operations. Silberstein and Chemero argue that, though these models are not - and cannot be mechanistic, they are still allow us to 'derive, predict and discover' (pg 964); in other words, they can serve as a guide to discovery (Chemero, 2009).

In this paper, we propose that it is possible to meaningfully decompose cognitive systems into real parts and processes, and that we therefore do not have to settle for DST models as our best explanations. The first step in getting to the relevant real parts and operations for a given cognitive

\footnotetext{
${ }^{2}$ Stating that psychological models do not typically contain real parts and operations is not the same as saying that they require a dualism. It is just that being physically realizable is not the same as knowing how particular parts and operations are physically realized, and the computational approach to psychology was driven by the former, rather than the latter (e.g. Goel, 2003).
} 
capacity is to identify the correct scale (Eronen, 2015) for explaining that capacity, as in the fermentation example. Our thesis is that for animal behaviour, the relevant scale is picked out by ecological information (Gibson, 1979). In the following sections we first explain what the ecological scale is and then show what information is available to observers at that scale. We will then work within the constraints imposed on us by the nature of ecological information to identify additional real components in ecological mechanistic explanations. Our goal is to show that the ecological approach grounds psychological explanations in the types of parts and operations that are amenable to dynamic causal mechanistic models.

\section{Perceptual Information \& the Ecological Scale}

Most of the environment is 'over there' (i.e. not in mechanical contact with our bodies). Somewhat inconveniently, in order to stay alive our behaviour must be shaped by these distal properties, so a central challenge for perceiving/acting organisms is acting with respect to things with which they are not in mechanical contact. We clearly manage this, so the question is how? The short answer is that organisms are immersed in various perceptual media (e.g. light for vision) and patterns within these media arrays act as remarkably good stand ins ${ }^{3}$ for properties in the environment. The key to this is the ability of patterns in energy media to specify dynamic properties of the world, which we explain in detail below.

\section{The World is Dynamic}

There are many different ways to describe objects and events in the world, but it is only at the level of dynamics that categories of objects and events can be uniquely identified (Bingham, 1995). This simply means that a comprehensive mathematical representation of some object or event will

\footnotetext{
${ }^{3}$ This notion of 'standing-in for' implies that information is serving as a representation. This is basically true, although it is not the kind of representation typically invoked in the cognitive sciences. We have analysed this implication in detail (Golonka \& Wilson, 2018). For the purposes of our current argument, it is only important to note that information is a very particular kind of mediating connection between organisms and properties of the environment.
} 
require units of time, length and mass (forces). A dynamical description of an event type is an equation that includes a specific set of variables placed in a particular relation to each other using operators such as addition, differentiation, etc. This equation describes the characteristic form a category of events take as it unfolds over space and time. A particular instance of an event type is specified by setting parameters on those variables. Two throws, for example, are both examples of the dynamic event category projectile motion even if they differ in their parameters (release angle, speed or height). In order to functionally interact with the world, an organism's behaviour must complement the current dynamical state of the world, otherwise it falls over, gets eaten, etc.

\section{Information is Kinematic}

Fortunately for us, these typically distant dynamics interact with various media (e.g., light, atmosphere) that we are also embedded in, and they are projected as patterns into those media. This projection is governed by ecological laws (Turvey et al, 1981), and through this interaction radiant light (for example) becomes structured ambient light (Gibson, 1979). The field of energy is no longer symmetrical; there are variations in density, intensity, etc that make each given view point unique. The field has become an array. Put simply, environmental properties structure various media in particular ways.

For structures in arrays to be really useful to organisms, they should be good stand-ins for the dynamical properties that cause them. The most meaningful way in which they can be "good" standins is if a particular structure in an energy array maps 1:1 with an environmental property of interest. The patterns projected into energy media are kinematic, not dynamic; a complete description doesn't require mass (Bingham, 1988). This means that kinematic information cannot be identical to dynamics. Fortunately, kinematic patterns can be specific (i.e., map 1:1) to dynamics (Runeson \& Frykholm, 1983; Turvey et al, 1981), meaning that they can serve as good stand-ins for properties of interest. This is ecological information. 
There is now extensive evidence that kinematic patterns in ambient arrays can and do specify dynamic properties, and that organisms can and do use the kinematic patterns as information for the underlying dynamics. We have no room here for an extensive review of this evidence, but we want to mention the major lines of research to support our claims here;

1. Dynamic Occlusion: when one surface goes out of view behind another, it creates a specific pattern in the optic array (progressive accretion and deletion of optical texture at the leading and trailing edges, respectively). People perceive the dynamical event 'occlusion' rather than an object ceasing to exist (Gibson et al, 1969; Kaplan, 1969) with powerful consequences on multiple object tracking (Scholl \& Pylyshyn, 1999).

2. Event Perception (Johansson et al, 1980): This work uses point light displays in which only kinematic information about the underlying dynamics is presented to an observer. These displays support perception of a wide range of both physical and social properties (size and weight but also gender, intent to deceive and vulnerability to attack; Blake and Shiffrar, 2007).

3. Optic Flow: Relative motion between an observer and the environment creates optic flow, which specifies both spatial and temporal elements of both self- (global flow) and other(local flow) motion (Warren, 2004). Research here focuses on the control of locomotion (e.g. Fajen \& Warren, 2003; Wilkie \& Wann, 2003) and interception (e.g. McBeath et al, 1995).

4. Non-Visual Information: While research on information is dominated by vision, there are examples that show how we can perceive dynamical properties acoustically (Button \& Davids, 2004; Gaver, 1993; Warren and Verbrugge, 1984; Warren et al, 1987). A related field is work on echolocation in bats and dolphins (Thomas et al, 2004), orca (Au et al, 2010), and humans (Kolarik et al, 2014).

5. No Information, No Perception: When information is available, functional and adaptive behaviours can be assembled to complement the specified dynamics. When information is not present, behaviour fails catastrophically. For example, friction between two surfaces 
does not exist ahead of them coming into contact and it therefore creates no information ahead of contact. Consequently, people are highly variable and dysfunctional when asked to make friction-related judgments ahead of acting (Joh et al, 2006, 2007) and friction-related injuries are common and reactive (Courtney et al, 2001).

The practical upshot of all this research is that higher-order kinematic patterns of motion created by and specific to dynamical events (i.e., ecological information variables) are an extraordinarily rich source of information about those dynamics. More importantly, humans and other animals are exquisitely sensitive to these patterns and can learn to use them as information (e.g. dogs, Shaffer et al, 2004; cats, Blake, 1993; pigeons, Dittrich et al, 1998; newborn chicks, Regolin, et al, 2000).

\section{Information is the Ground Floor Scale for Behavioural Mechanistic Explanations}

A key element of this kinematic specification of dynamics analysis is that we use kinematic patterns to stand-in for dynamics. So why ground our mechanistic models of behaviour in ecological information, and not dynamical properties?

The key here is that kinematic, ecological information is our only point of contact with distal properties of the environment. Just as our response to a mechanical event depends on the form of the force being applied, our response to the perception of a dynamical event depends on the form of the information enabling that perception. From the point of view of the organism, behaviour begins with information. Therefore, information is where will ground our explanations, in exactly the same way that biochemicals identify the correct scale to ground explanations of fermentation.

The next two sections explore two other behaviourally relevant components that are constrained by / constrain the use of information. The first is the processes of action coordination (selection) and control, and the second is the activity of the nervous system. The ecological analysis of how these two components operate provide additional support for the claim that information is the correct scale at which to ground our explanations. 


\section{Task Specific Devices, Caused by Information}

The job of the action system is functional coordination with the environment. Action systems are massively complex, and, since Bernstein, researchers have tried to uncover how they can be suitably constrained to organise functional, adaptive behaviour. "Organising behaviour" means arranging the components of an action system so that the dynamics of the resulting arrangement complement the task dynamics (e.g., behaviourally relevant environmental properties).

Any component that can change state is a degree of freedom, something whose state must be set, or controlled. Even simple action systems contain many degrees of freedom, which creates a significant control problem, known (handily enough) as the degrees of freedom problem (Bernstein, 1967). When you have more degrees of freedom than required to solve the task, the system is redundant; you can complement task dynamics in more than one way. This provides essential flexibility ('the bliss of motor abundance'; Latash, 2012), but having a large space of possible actions means you must constrain the space in task relevant ways to be able to quickly and efficiently select the appropriate actual action.

Historically, there are two types of solution to the problem (Rosenbaum et al, 1996) but both fail to help us here. The first is that the action system 'freezes out' some degrees of freedom, via biomechanical linkages (e.g. Alexander, 1991) or softly assembled synergies (Latash, 2008; Turvey, 1990). But which degrees of freedom to freeze, and why? The second is via cost functions (e.g. minimising jerk; Flash \& Hogan, 1985), which guides selection to the 'cheapest' solution. But because costs are task specific (Rosenbaum et al, 1996), we're still left asking which cost function, and why? Bingham (1988) examined this problem in detail and identified that both these proposals start in the wrong place, with the full, unfrozen system. Every subsystem has a huge number of degrees of freedom, their own dynamic characteristics, and is embedded in the complex dynamics of the local environment. The unfrozen system is simply too big of a place to start (both for the organism and the scientist studying that organism). Bingham proposed that the only thing that can constrain 
action to functional, task-relevant solutions are the task demands themselves. Task demands are perceived via information. Consequently, ecological information specifying task dynamics constrains action coordination and control to the space of task-relevant solutions.

Because information is created by task dynamics, it only exists when those dynamics do; behaviour organised with respect to information will therefore have to be assembled in real time (while the task and therefore the information is present). Behaviour is task-specific and a given solution is a task-specific device (TSD; Bingham, 1988).

One set of environmental properties are called affordances (Gibson, 1979; see Wilson et al, 2018 for a review of an explicitly task dynamical analysis of affordances in the context of targeted throwing). Affordances are action relevant dynamical properties ${ }^{4}$ of objects in the context of tasks; not 'how large is that object?' but 'is it graspable by me?' or 'is it step-overable by me?'. TSDs assembled with respect to the perception of these intrinsically action-scaled properties means the space of possible actions is immediately and appropriately constrained by the task at hand. Bingham (1988) also includes advice and guidance on researching this perception-action problem space.

\section{Neural Activity, Caused by Information}

Information constrains the formation of task-specific action systems that can complement the specified task dynamics (affordances). But how are TSD components coupled to information, and what happens to the informational structure in energy arrays en route from perceptual receptors to other bodily systems? The answer, of course, lies in the nervous system, our next component.

The ecological approach does not (yet) have much to say about the brain. However, we have argued extensively elsewhere (Golonka \& Wilson, 2018) that the form of nervous system activity is

\footnotetext{
${ }^{4}$ There is a seemingly endless debate about whether affordances are best formalized as dispositional properties of the environment (Scarantino, 2003; Turvey, 1992) or as relations in the animal-environment system (e.g. Chemero, 2009). We treat them here as dispositional properties because we believe it is a more scientifically useful formulation that remains true to Gibson's original intent for the concept and enables them to be perceptible (Wilson, 2018b).
} 
constrained by interactions with information. Recent ecological neuroscience research that shows neural activity preserves, rather than transforms, the temporal structure present in information (Agyei et al, 2015; Magrassi et al, 2015; van der Meer et al, 2012; van der Weel \& van der Meer, 2009). This provides a very different way to interpret neural activity.

Cognitive neuroscience interprets neural activity with respect to cognitive level theories, but as noted these are functional, 'how-possibly' models. There is therefore no guarantee that the cognitive capacities being investigated ('attention', 'memory', etc) will map onto material level components, even if those cognitive level descriptions capture some regularity in behaviour (Cummins, 2000). This is one reason why cognitive neuroscience is often concerned with neural correlates of cognitive capacities.

The neural correlates approach faces two problems in identifying real neural components, namely neural reuse (Anderson, 2014) and neural degeneracy (e.g. Edelman \& Gally, 2001). Neural reuse is when neural circuits originally dedicated to one capacity are recuited to achieve new capacities, without losing their original function. Brain regions do not specialise solely in particular types of cognitive tasks (e.g. memory tasks), but instead they operate across domains that don't have any obvious cognitive-level commonality. Neural degeneracy presents the opposite problem; a single capacity may be expressed through many different neural implementations. These are both features, not bugs; they enable flexible and adaptive behaviour in the face of varying task demands and injury to the nervous system. We have just argued above that the related problems in action coordination and control can only be meaningfully constrained by information; we propose the same is true of neural coordination and control, for exactly the same reason (Golonka \& Wilson, 2018).

In contrast to the correlates approach, the ecological components described so far firmly constrain what the nervous system must accomplish in order to support a specific behaviour. Specifically, the informational and task dynamical analysis identifies which components are available to be connected, and whether behaviour shows evidence of structure not fully explained by information. 
This places strict but useful limits on our expectations for and interpretations of neural activity during a behaviour. One of the biggest strengths of this approach is the ability to use a common mathematical language throughout. For instance, the dynamic description of task-relevant properties can be related to the kinematic description of information variables, which can be related a time series of neural activity, which can be related to a dynamic description of the action system. Therefore, our first implication for neuroscience is that the interpretation of neural activity can best be meaningfully constrained by what we already know about the form of the behavioural mechanism built of the components described above (Golonka \& Wilson, 2018) ${ }^{5}$.

There is clearly much more empirical work to do here, but as noted it has begun and, we argue, it will enable the development of mechanistic explanations of the neural level if it is grounded in our ecological analysis (see Wilson \& Golonka, 2018 for more).

\section{A Mechanistic Model of Coordinated Rhythmic Movement}

We have so far developed a framework that can, in principle, support the development of dynamic causal mechanistic models of behaviour. Environmental properties create ecological information which causes skilled action systems to form task specific devices, the functioning of which enables an organism to complement the dynamics of the environment. We have also proposed how studying behavioural mechanisms at this scale can constrain the analysis of the nervous system, using the common language of dynamical systems theory. It's now time to examine a specific example of the kind of model this approach can produce as a proof-of-concept for our approach, to demonstrate that (contra Chemero and colleagues), it is possible to meaningfully decompose and localise real components of cognitive systems and model the results as a mechanism.

\footnotetext{
${ }^{5}$ Conceptualising the role of the nervous system as enabling ongoing, context sensitive coordination of bodily systems with the perceived environment also connects nicely to the excellently named Skin-Brain Thesis of nervous system evolution (Keijzer, Duijn \& Lyon, 2013).
} 
This section will analyse the development and the current form of an ecological, dynamical systems model of coordinated rhythmic movement developed by Geoffrey Bingham and colleagues (Bingham, 2001, 2004a, b; Snapp-Childs, Wilson \& Bingham, 2011). This model is a causal mechanistic model designed to replace an influential but non-mechanistic account, the famous Haken-Kelso-Bunz model (HKB; Haken, Kelso \& Bunz, 1985). We will briefly sketch out the key empirical phenomena, and then detail how the empirical programme of Bingham and colleagues decomposed the system and localised many of the components. Finally, we show how mathematical representations of these components have been implemented in a mechanistic model of the phenomena. This programme lives up to the challenge posed by Bechtel and Abrahamsen (2010) and the result is a mechanistic model of a behavioural system. This model successfully explains all the known phenomena, has made novel predictions which have then been empirically verified and, even though it does not yet explicitly handle learning, it has constrained and informed multiple learning studies which are even now feeding back into an expansion of the model. We have considered this model before in terms of the benefits of theory-driven science (Golonka \& Wilson, 2012) but here we want to explicitly hold this model up as an exemplar of the kind of mechanistic explanations that are possible with our approach.

\section{The Task Dynamics of Coordinated Rhythmic Movement}

In its simplest form, coordinated rhythmic movement involves moving two things in parallel rhythmic oscillations that are placed in a particular relation (a specific relative phase). The oscillators can be limbs belonging to the same or different people (bimanual coordination) or one of them can even be a simulation on a computer screen (unimanual coordination). So long as at least one of the oscillators is being controlled by a person, however, the following phenomena apply.

If the oscillators move so that they are doing the same thing at the same time (e.g. both flexing then extending) then they are moving at $0^{\circ}$ mean relative phase. If they are moving so that they are doing the opposite thing at the same time (e.g. one flexing, the other extending) then they are moving at 
$180^{\circ}$ mean relative phase. All possible coordinations can be uniquely identified with a mean relative phase, e.g. $90^{\circ}$ is where the oscillators are out of phase by a quarter cycle.

It turns out this simple task has some intriguing structure to it:

1. Without at least some training, the only two coordinated rhythmic movements that people can stably produce are $0^{\circ}$ and $180^{\circ}$. All other coordinations are produced with large errors (high relative phase SD) and people have a tendency to switch from the intended relative phase into either $0^{\circ}$ or, less frequently, $180^{\circ}$. They can all be learned, however; jazz drummers are a possibility!

2. $0^{\circ}$ is more stable than $180^{\circ}$. As the required frequency is increased, movements at both $0^{\circ}$ and $180^{\circ}$ become less stable (increasing relative phase SD) but more so at $180^{\circ}$. At a certain frequency (bimanual: $\sim 3-4 \mathrm{~Hz}$, unimanual: $\sim 1.5 \mathrm{~Hz}$ ) people become unable to maintain $180^{\circ}$ and (under a non-interference instruction) transition to $0^{\circ}$.

These characteristics were first identified by Cohen $(1971)$ and Yaminishi et al $(1979,1980)$ and then studied in detail by Kelso (Kelso, 1995). The HKB model (Haken et al, 1985) produces these characteristics using an abstract potential function, which describes the amount of 'energy' required to obtain a given relative phase. The model has been extremely influential, but Kelso never intended it to be anything like a causal mechanistic model (Kelso \& Engstrom, 2006). That said, the HKB model motivated a large number of experiments that revealed many of the key nonlinear dynamical features of coordinated rhythmic movement.

\section{The Perception-Action Approach to Coordinated Rhythmic Movement}

The task dynamics of bimanual coordinated rhythmic movement is two oscillating human limbs moving so as to maintain a mean relative phase with respect to one another. Maintaining any state entails control, and control entails perception; relative phase must be perceived. The dynamic environmental property of interest is relative phase, the dynamic behaviour to be explained is the 
pattern of stable coordinations between the two limbs, and these are connected via a kinematic perceptual variable specifying relative phase.

\section{The Dynamics of Rhythmically Moving Limbs}

Two perturbation studies (Kay, et al 1987, 1991) demonstrated that a rhythmically moving human limb exhibits five specific properties which identify the kind of dynamical device it is. The two key properties are limit cycle stability and phase resetting. If you perturb a rhythmically moving limb (e.g. forcing it to briefly speed up or slow down) it settles back down into a periodic motion dynamically described as a limit cycle. It also settles back onto the limit cycle at a phase that is different from where it would be at that time if it hadn't been perturbed. Limit cycles mean that the overall dynamic of the limb is nonlinear; phase resetting means this dynamic is autonomous (phase is not an explicit function of time, but of the oscillators' own behaviour). The other three properties are an inverse frequency/amplitude relation, a direct frequency/velocity relation, and a rapid relaxation time post-perturbation, independent of frequency. These are emergent properties of the dynamic and are not directly imposed by components that have this feature as their purpose. Kay et al (1987) combined two well-known systems (the Rayleigh and the van der Pol oscillators) into a hybrid oscillator that behaved this way; it fits the data, but it is not a representation of an actual oscillating human limb.

Bingham (2004a) instead started with the $\lambda$-model of actual human limbs in which movements are generated by controlling the equilibrium point of a damped mass-spring (Feldman, 1986). A massspring is literally a mass bouncing on the end of a spring; a damped one includes friction and so, unless driven, it will eventually come to rest at an equilibrium point where the pull of the mass is balanced by the pull of the spring. Feldman's hypothesis is that a) human limbs are softly assembled into controllable synergies that act as damped-mass springs, and that b) you could easily move and control such a synergy by simply controlling the equilibrium point (a single degree of freedom). There is now extensive neurophysiological and behavioural evidence for the $\lambda$-model as the 
mechanism used to enable cheap and stable control of limbs (Feldman, 2011) so this is the appropriate dynamical characterisation of human limbs in this task ${ }^{6}$.

The basic form of a damped mass spring is

$$
\ddot{x}+b \dot{x}+k x=0
$$

where $x, \dot{x}$ and $\ddot{x}$ are state variables (position, velocity and acceleration) and $b$ and $k$ are parameters (damping and stiffness). Such a damped mass-spring eventually comes to rest because it is equal to a static value (here, 0 ). You keep such a spring moving by driving it (setting the equation to be equal to a value that changes over time). If the driver's value changes as a function built only from the state variables (rather than as a function of time) then the dynamic is autonomous.

Bingham (2004a, b) proposed that limbs engaged in rhythmic movements are phase driven damped mass-springs. Specifically, the model is

$$
\begin{gathered}
\ddot{x}+b \dot{x}+k x=c \sin [\Phi] \\
\text { where } \Phi=\arctan \left[\frac{\dot{x_{n}}}{x}\right], \dot{x}_{n}=\frac{\dot{x}}{\sqrt{k}}, \mathrm{k}=k_{i}+\gamma\left|e_{t} e_{i}\right|, e_{n}=\sqrt{v_{n}^{2}+x^{2}} \text { and } c=c(k)
\end{gathered}
$$

$\Phi$ is the phase angle, which changes over time but not as a function of time. It is composed solely of (real) state variables. The stiffness, $k$, varies in proportion to the perceived deviation from the limit cycle $\left(e_{n}\right.$ is the radius of the limit cycle on the phase diagram; perturbations of the limb move the trajectory off the cycle and change the current value of that radius). $c$ scales the amplitude of the limit cycle attractor for a given movement. We can now take two of these oscillators and couple them together via the perception of their relative phase.

\footnotetext{
${ }^{6}$ It would make a nice extension of Bingham's research to explicitly model the neural scale of control during this task using Feldman's equilibrium point work. Right now, Bingham's behavioural scale model is the equivalent of the biochemical level analysis of fermentation. It is a necessary step to guide decomposition and localisation work but critically it is one that still trades in real components; limb dynamics in this task are literally those of a damped mass-spring and it's those dynamics that matter to explaining the phenomena of interest.
} 


\section{The Perception of Relative Phase}

Being able to maintain a mean relative phase implies being able to perceive relative phase. Bingham set about identifying the kinematic information variable(s) specifying relative phase.

Schmidt et al (1990) showed that the basic HKB phenomena persist when the limbs being coordinated belonged to two different people coordinating via vision. Bingham et al (1999) therefore presented participants with coordinated rhythmic movements in the form of a simple point light display moving side-to-side or in depth (using both simulated movements and the kinematics from Schmidt et al). Participants judged coordination stability or phase variability in these displays (see also Zaal et al, 2000). The results were that the HKB pattern emerged, even with no movement required. $0^{\circ}$ was judged as maximally stable and the various levels of phase variability were clearly discriminated there. $180^{\circ}$ was judged as stable but less so, and phase variability discrimination was also poorer, while $90^{\circ}$ was judged to be maximally unstable and the added variability was not discriminated at all. The effects of frequency also appear in the judgment task, both visually (Bingham et al, 2001) and proprioceptively (Wilson et al, 2003). The symmetry between relative phases that is the HKB pattern is broken at the level of information, not movement dynamics.

Wilson et al (2005a) then reconnected these perceptual results to the movement task and showed that movement stability followed perceptual stability: movements at a variety of relative phases were stabilised by $0^{\circ}$ visual feedback, and destabilised by non- $0^{\circ}$ visual feedback. Wilson et al (2010) showed that training people to become expert perceivers of $90^{\circ}$ led to immediate improvement in the production of $90^{\circ}$, with no training on that movement. Snapp-Childs et al (2015) showed that movement training leads to improved perceptual thresholds with no training on the perceptual task. These demonstrated a causal link between the perception and production of coordinated rhythmic movements. 
There is therefore extensive evidence that the form of coordinated rhythmic movement behaviour comes from the perception of relative phase. This evidence was then used constrain the form of the perceptual coupling to implement in the model. Although it would be possible to implement the coupling using an abstract component that simply successfully matched the data, Bingham and colleagues used the empirical evidence to guide the search for a specific variable that matched the following criteria. The information variable must be kinematic, it must specify relative phase, it must be made of state variables (to preserve autonomy), it must break the symmetry between relative phases in a manner that matches the phenomena catalogued above, and it must be detectable by both vision and proprioception.

There was some prior evidence that relative direction of motion was important to the perception of relative phase. The HKB effects disappear when the movements are orthogonal to each other (i.e. when relative direction is not uniquely defined; Swinnen et al, 1998; Wimmers et al, 1992), and coordinated rhythmic movements are most stable with parallel feedback displays (Bogaerts et al, 2003). Learning a novel coordination only transfers to that coordination's symmetry partner (e.g. learning $90^{\circ}$ only transfers to $270^{\circ}$ ); these states are identical at the level of relative direction and only differ in which limb leads or lags (Zanone \& Kelso, 1992, 1997). Finally, Wilson et al (2005b) showed that orthogonal components of motion simply added noise to coordination and had no relative phase-specific effects.

\section{A Mechanistic, Perception-Action Model of Coordinated Rhythmic Movement}

With all the evidence in place, Bingham $(2001,2004 a, b)$ proposed that bimanual coordinated rhythmic movement consists of two phase-driven damped mass-springs, each driven by the perceived phase of the other with this driver modified by the perceived relative phase. He hypothesised that relative phase is perceived using the relative direction of movement (rho, P), conditioned by the relative speed. The model takes the form

$$
\ddot{x}_{1}+b \dot{x}_{1}+k x_{1}=c \sin \left(\Phi_{2}\right) P_{i j}
$$




$$
\ddot{x}_{2}+b \dot{x}_{2}+k x_{2}=c \sin \left(\Phi_{1}\right) P_{j i}
$$

where $P_{i j}=\operatorname{sgn}\left(\sin \left(\Phi_{1}\right) \sin \left(\Phi_{2}\right)+\alpha\left(\dot{x}_{i}-\dot{x}_{j}\right) N_{t}\right.$

Judgments are modelled by integrating perceived relative phase over time (Bingham, 2004a) while unimanual coordination is modelled by simply setting one driver to 0 (Snapp-Childs et al, 2011).

Relative direction of motion behaves in a manner that maps directly onto the HKB coordination phenomena. At $0^{\circ}$, relative direction is always the same (in terms of the behaviour of the optic array, all the local flow is common motion); this is maximally stable and easy to detect. At $180^{\circ}$, relative direction is always different (all the local flow is relative motion). This is easy to detect, but less so than all common motion. At $90^{\circ}$, relative direction is maximally variable (half the time the same, half the time different, and constantly switching) making it intrinsically unstable, difficult to detect and therefore unable to support stable behaviour.

The analysis of the task that underpins the model also revealed that behaviour is controlled with respect to information, and not directly with respect to the dynamics of relative phase. This resolves a long-standing question in the field about rates of learning. Zanone \& Kelso (1994) predicted (on the basis of their attractor dynamical analysis) that learning novel relative phases close to $0^{\circ}$ should be more difficult than learning those close to $180^{\circ}$. This is because the attractor at $0^{\circ}$ is stronger and would therefore compete more effectively against the to-be-learned relative phase. Two studies found that the opposite is true; it's easier to learn novel coordinations near $0^{\circ}$ (Fontaine et al, 1997; Wenderoth et al, 2002, who explicitly noted that this result best makes sense in light of the perceptual results). Movement is not stable at $0^{\circ}$ because there is an attractor there, it is stable because $0^{\circ}$ and the relative phases around it are clearly perceived and discriminated because the optic flow is dominated by common motion. This clear perception then supports stable action.

The current version of the model represents many of the real components involved in the production and judgement of bimanual or unimanual coordinated rhythmic movements. The model 
is the result of an empirical research programme very similar in form to work on circadian rhythms used by Bechtel and Abrahamsen (2010) as an exemplar of the kind of programme cognitive science should adopt in order to develop mechanisms. The model accounts for existing phenomena and predicted several details of the mechanism that have since been empirically confirmed (movement stability is a function of perceptual stability, Wilson et al, 2010; relative direction is the information, Wilson \& Bingham, 2008; relative speed acts as a noise term, Snapp-Childs et al, 2011). There remains much to do, but the model also provides the framework for the next round of mechanistic empirical research, on learning (e.g. Leach et al, 2019; Snapp-Childs et al, 2015), changes in the perception-action system with ageing (Coats et al, 2013, 2014; Ren et al, 2015) and the interplay between visual and haptic perception of relative phase (Pickavance et al, 2018).

\section{Summary}

Bechtel and Abrahamsen (2010) detailed what it takes to develop truly mechanistic explanations of dynamical phenomena and challenged cognitive science to find a way to develop such explanations of cognition and behaviour. The goal of this paper was to rise to that challenge. Dynamic causal mechanistic explanation and modelling requires a clear understanding of the real parts and processes underlying the phenomenon of interest, as well as a suitable scale from which to begin this analysis. Here we have argued that the ecological approach to perception-action provides the appropriate scale for understanding human behaviour (the ecological scale) and that the identification of this scale leads to the use of real components (environmental properties, kinematic information specific to these properties, action systems) in ecological research programmes. We demonstrated this by reviewing the empirical programme and resulting model of coordinated rhythmic movement developed by Bingham and colleagues, which acts as a proof of concept that the ecological approach can lead to the development of mechanistic explanations.

The cognitive sciences do not have to take a mechanistic turn, and our goal has not been to force a mechanistic approach onto an ecological approach that may not suit such a turn. Quite the contrary; 
we simply identified a match between how philosophers of science describe mechanisms and the ontology of the ecological approach, which contains real parts and processes. Bingham's program of research then shows that you can, in fact, do meaningful decomposition and localization research at the behavioural level, and when you do, you gain the many explanatory benefits of a mechanistic approach. Bingham's model works better as an explanation than dynamical models such as the HKB, for example. So our proposal is simply this: if we do want mechanistic accounts in cognitive science, the ecological approach can support these, and they can be productive and successful. 


\section{References}

Agyei, S. B., Holth, M., Weel, F. R., \& Meer, A. L. (2015). Longitudinal study of perception of structured optic flow and random visual motion in infants using high-density EEG. Developmental science, 18(3), 436-451.

Alexander, R. M. (2006). Dinosaur biomechanics. Proceedings of the Royal Society of London B: Biological Sciences, 273(1596), 1849-1855.

Anderson, M. L. (2014). After phrenology: Neural Reuse and the Interactive Brain. Cambridge, MA: MIT Press.

Au, W. W., Horne, J. K., \& Jones, C. (2010). Basis of acoustic discrimination of chinook salmon from other salmons by echolocating Orcinus orca. The Journal of the Acoustical Society of America, 128(4), 2225-2232.

Bechtel, W. (2008a). Mental mechanisms: Philosophical perspectives on cognitive neuroscience. Taylor \& Francis.

Bechtel, W. (2008b). Mechanisms in cognitive psychology: What are the operations?. Philosophy of Science, 75(5), 983-994.

Bechtel, W., \& Abrahamsen, A. (2010). Dynamic mechanistic explanation: Computational modeling of circadian rhythms as an exemplar for cognitive science. Studies in History and Philosophy of Science Part A, 41(3), 321-333.

Bechtel, W., \& Richardson, R. C. (2010). Discovering complexity: Decomposition and localization as strategies in scientific research. MIT press.

Bernstein, N. A. (1967). The coordination and regulation of movement. London.

Bingham, G.P. (1988). Task specific devices and the perceptual bottleneck. Human Movement Science, 7, 5-264. 
Bingham, G. P. (1995). Dynamics and the problem of visual event recognition. Mind as motion: Explorations in the dynamics of cognition, 403-448.

Bingham, G.P. (2001). A perceptually driven dynamical model of rhythmic limb movement and bimanual coordination. Proceedings of the 23rd Annual Conference of the Cognitive Science Society, (pp. 75-79). Hillsdale, N.J., LEA Publishers.

Bingham, G.P. (2004a). A perceptually driven dynamical model of bimanual rhythmic movement (and phase perception). Ecological Psychology, 16(1), 45-53.

Bingham, G.P. (2004b). Another timing variable composed of state variables: Phase perception and phase driven oscillators. In H. Hecht \& G.J.P. Savelsbergh (Eds.) Theories of Time-to-Contact. Boston: MIT Press.

Bingham, G. P., Schmidt, R. C., \& Zaal, F. T. (1999). Visual perception of the relative phasing of human limb movements. Perception \& psychophysics, 61(2), 246-258.

Bingham, G. P., Zaal, F. T., Shull, J. A., \& Collins, D. R. (2001). The effect of frequency on the visual perception of relative phase and phase variability of two oscillating objects. Experimental Brain Research, 136(4), 543-552.

Blake, R. (1993). Cats perceive biological motion. Psychological Science, 4(1), 54-57.

Blake, R., \& Shiffrar, M. (2007). Perception of human motion. Annual review of psychology, 58.

Bogaerts, H., Buekers, M. J., Zaal, F. T., \& Swinnen, S. P. (2003). When visuo-motor incongruence aids motor performance: the effect of perceiving motion structures during transformed visual feedback on bimanual coordination. Behavioural Brain Research, 138(1), 45-57.

Button, C., \& Davids, K. (2004). Acoustic information for timing. Time-to-contact. Amsterdam: Elsevier, 355-369. 
Charles, E. P., Wilson, A. D., \& Golonka, S. (2014). The most important thing neuropragmatism can do: Providing an alternative to 'cognitive' neuroscience. In Pragmatist Neurophilosophy: American Philosophy and the Brain, (eds. Shook, J., \& Solymosi, T). Bloomsbury Academic.

Chemero, A. (2009). Radical embodied cognitive science. MIT press.

Chemero, A., \& Silberstein, M. (2008). After the Philosophy of Mind: Replacing Scholasticism with Science. Philosophy of Science, 75(1), 1-27.

Coats, R. O., Snapp-Childs, W., Wilson, A. D., \& Bingham, G. P. (2012). Perceptuo-motor learning rate declines by half from 20s to 70/80s. Experimental Brain Research, 225(1), 75-84.

Coats, R. O., Wilson, A. D., Snapp-Childs, W., Fath, A. J., \& Bingham, G. P. (2014). The 50s cliff: Perceptuo-motor learning rates across the lifespan. PLOSOne, 9(1), e85758. DOI: 10.1371/journal.pone.0085758.

Cohen, L. (1971). Synchronous bimanual movements performed by homologous and nonhomologous muscles. Perceptual and motor skills, 32(2), 639-644.

Courtney, T. K., Sorock, G. S., Manning, D. P., Collins, J. W., \& Holbein-Jenny, M. A. (2001). Occupational slip, trip, and fall-related injuries-can the contribution of slipperiness be isolated?. Ergonomics, 44(13), 1118-1137.

Cummins, R. (2000). How does it work?" versus" what are the laws?": Two conceptions of psychological explanation. Explanation and cognition, 117-144.

Craver, C. F. (2007). Explaining the brain: Mechanisms and the mosaic unity of neuroscience. Oxford University Press.

Dittrich, W. H., Lea, S. E., Barrett, J., \& Gurr, P. R. (1998). Categorization of natural movements by pigeons: Visual concept discrimination and biological motion. Journal of the experimental analysis of behavior, 70(3), 281-299. 
Edelman, G. M., \& Gally, J. A. (2001). Degeneracy and complexity in biological systems. Proceedings of the National Academy of Sciences, 98(24), 13763-13768.

Eronen, M. I. (2015). Levels of organization: a deflationary account. Biology \& Philosophy, 30(1), 3958.

Fajen, B. R., \& Warren, W. H. (2003). Behavioral dynamics of steering, obstacle avoidance, and route selection. Journal of Experimental Psychology: Human Perception and Performance, 29, 343362.

Feldman, A. G. (1986). Once more on the equilibrium-point hypothesis ( $\lambda$ model) for motor control. Journal of motor behavior, 18(1), 17-54.

Feldman, A. G. (2011). Space and time in the context of equilibrium-point theory. Wiley Interdisciplinary Reviews: Cognitive Science, 2(3), 287-304.

Flash, T., \& Hogan, N. (1985). The coordination of arm movements: an experimentally confirmed mathematical model. Journal of neuroscience, 5(7), 1688-1703.

Fontaine, R. J., Lee, T. D., \& Swinnen, S. P. (1997). Learning a new bimanual coordination pattern: Reciprocal influences of intrinsic and to-be-learned patterns. Canadian Journal of Experimental Psychology, 51(1), 1.

Gaver, W. W. (1993). What in the world do we hear?: An ecological approach to auditory event perception. Ecological psychology, 5(1), 1-29.

Gibson, J. J. (1979). The ecological approach to visual perception. Boston: Houghton Mifflin.

Gibson, J. J., Kaplan, G. A., Reynolds, H. N., \& Wheeler, K. (1969). The change from visible to invisible. Perception \& Psychophysics, 5(2), 113-116.

Glennan, S. S. (1996). Mechanisms and the nature of causation. Erkenntnis, 44(1), 49-71. 
Goel, V. (2005). Cognitive neuroscience of deductive reasoning. The Cambridge handbook of thinking and reasoning, 475-492.

Golonka, S. (2015). Laws and conventions in language-related behaviors. Ecological Psychology, 27(3), 236-250.

Golonka, S., \& Wilson, A. D. (2012). Gibson's ecological approach-a model for the benefits of a theory driven psychology. Avant, 3(2), 40-53.

Golonka, S., \& Wilson, A. D. (2018) Ecological Representations. bioRxiv 058925; doi: https://doi.org/10.1101/058925

Haken, H., Kelso, J. S., \& Bunz, H. (1985). A theoretical model of phase transitions in human hand movements. Biological Cybernetics, 51(5), 347-356.

Joh, A. S., Adolph, K. E., Campbell, M. R., \& Eppler, M. A. (2006). Why walkers slip: Shine is not a reliable cue for slippery ground. Perception \& Psychophysics, 68(3), 339-352.

Joh, A. S., Adolph, K. E., Narayanan, P. J., \& Dietz, V. A. (2007). Gauging possibilities for action based on friction underfoot. Journal of Experimental Psychology: Human Perception and Performance, 33(5), 1145.

Johansson, G., Hofsten, C. V. H., \& Jansson, G. (1980). Event perception. Annual review of psychology, 31(1), 27-63.

Kaplan, G. A. (1969). Kinetic disruption of optical texture: The perception of depth at an edge. Perception \& Psychophysics, 6(4), 193-198.

Kay, B. A., Kelso, J. A., Saltzman, E. L., \& Schöner, G. (1987). Space-time behavior of single and bimanual rhythmical movements: Data and limit cycle model. Journal of Experimental Psychology: Human Perception and Performance, 13(2), 178. 
Kay, B. A., Saltzman, E. L., \& Kelso, J. A. (1991). Steady-state and perturbed rhythmical movements: A dynamical analysis. Journal of Experimental Psychology: Human Perception and Performance, 17(1), 183.

Keijzer, F. (2006). Differentiating Animality from Agency Towards a Foundation for Cognition. In Proceedings of the Annual Meeting of the Cognitive Science Society (Vol. 28, No. 28).

Keijzer, F., Van Duijn, M., \& Lyon, P. (2013). What nervous systems do: early evolution, input-output, and the skin brain thesis. Adaptive Behavior, 21(2), 67-85.

Kelso, J. S. (1995). Dynamic patterns: The self-organization of brain and behavior. MIT press.

Kelso, J. S., Engstrom, D. A., \& Engstrom, D. (2006). The complementary nature. MIT press.

Kolarik, A. J., Cirstea, S., Pardhan, S., \& Moore, B. C. (2014). A summary of research investigating echolocation abilities of blind and sighted humans. Hearing research, 310, 60-68.

Latash, M. L. (2008). Synergy. Oxford University Press.

Latash, M. L. (2012). The bliss (not the problem) of motor abundance (not redundancy). Experimental brain research, 217(1), 1-5.

Leach, D. P., Kolokotroni, Z., \& Wilson, A. D. (2019, April 1). Perceptual Information Supports Transfer of Learning in Coordinated Rhythmic Movement. https://doi.org/10.31234/osf.io/c8qvp

Magrassi, L., Aromataris, G., Cabrini, A., Annovazzi-Lodi, V., \& Moro, A. (2015). Sound representation in higher language areas during language generation. Proceedings of the National Academy of Sciences, 112(6), 1868-1873.

McBeath, M. K., Shaffer, D. M., \& Kaiser, M. K. (1995). How baseball outfielders determine where to run to catch fly balls. Science, 268(5210), 569-573. 
Pickavance, J., Azmoodeh, A., \& Wilson, A. D. (2018). The effects of feedback format, and egocentric \& allocentric relative phase on coordination stability. Human Movement Science, 59, 143-152.

Regolin, L., Tommasi, L., \& Vallortigara, G. (2000). Visual perception of biological motion in newly hatched chicks as revealed by an imprinting procedure. Animal Cognition, 3(1), 53-60.

Ren, J., Huang, S., Zhang, J., Zhu, Q., Wilson, A. D., Snapp-Childs, W. \& Bingham, G. P. (2015). The 50s Cliff: A Decline in perceptuo-motor learning, not a deficit in visual motion perception. PLoS ONE 10(4): e0121708. doi:10.1371/journal.pone.0121708.

Rosenbaum, D. A., Meulenbroek, R. G., \& Vaughan, J. (1996). Three approaches to the degrees of freedom problem in reaching. In Hand and brain (pp. 169-185).

Runeson, S., \& Frykholm, G. (1983). Kinematic specification of dynamics as an informational basis for person-and-action perception: expectation, gender recognition, and deceptive intention. Journal of Experimental Psychology: General, 112(4), 585.

Scarantino, A. (2003). Affordances explained. Philosophy of Science, 70(5), 949-961.

Schmidt, R. C., Carello, C., \& Turvey, M. T. (1990). Phase transitions and critical fluctuations in the visual coordination of rhythmic movements between people. Journal of experimental psychology: human perception and performance, 16(2), 227.

Scholl, B. J., \& Pylyshyn, Z. W. (1999). Tracking multiple items through occlusion: Clues to visual objecthood. Cognitive psychology, 38(2), 259-290.

Shaffer, D. M., Krauchunas, S. M., Eddy, M., \& McBeath, M. K. (2004). How dogs navigate to catch Frisbees. Psychological Science, 15(7), 437-441.

Silberstein, M., \& Chemero, A. (2013). Constraints on localization and decomposition as explanatory strategies in the biological sciences. Philosophy of Science, 80(5), 958-970. 
Snapp-Childs, W., Wilson, A.D. \& Bingham, G.P. (2011). The stability of rhythmic movement coordination depends on relative speed: The Bingham model supported. Experimental Brain Research, 215, 89-100.

Snapp-Childs, W., Wilson, A. D., \& Bingham, G. P. (2015). Transfer of learning between unimanual and bimanual rhythmic movement coordination: transfer is a function of the task dynamic. Experimental brain research, 233(7), 2225-2238.

Stepp, N., Chemero, A., \& Turvey, M. T. (2011). Philosophy for the rest of cognitive science. Topics in Cognitive Science, 3(2), 425-437.

Swinnen, S. P., Jardin, K., Verschueren, S., Meulenbroek, R., Franz, L., Dounskaia, N., \& Walter, C. B. (1998). Exploring interlimb constraints during bimanual graphic performance: effects of muscle grouping and direction. Behavioural brain research, 90(1), 79-87.

Thomas, J. A., Moss, C. F., \& Vater, M. (Eds.). (2004). Echolocation in bats and dolphins. University of Chicago Press.

Turvey, M. T. (1990). Coordination. American psychologist, 45(8), 938.

Turvey, M. T. (1992). Affordances and prospective control: An outline of the ontology. Ecological psychology, 4(3), 173-187.

Turvey, M. T., Shaw, R. E., Reed, E. S., \& Mace, W. M. (1981). Ecological laws of perceiving and acting: In reply to Fodor and Pylyshyn (1981). Cognition, 9(3), 237-304.

Van Der Meer, A. L., Svantesson, M., \& Van Der Weel, F. R. (2012). Longitudinal study of looming in infants with high-density EEG. Developmental Neuroscience, 34(6), 488-501.

Van der Weel, F. R., \& Van der Meer, A. L. (2009). Seeing it coming: infants' brain responses to looming danger. Naturwissenschaften, 96(12), 1385-1391. 
Warren, W. H. (2004). Optic flow. In L. M. Chalupa \& J. S. Werner (Eds.), The visual neurosciences, v. II (pp. 1247-1259). Cambridge, MA: MIT Press.

Warren Jr, W. H., Kim, E. E., \& Husney, R. (1987). The way the ball bounces: visual and auditory perception of elasticity and control of the bounce pass. Perception, 16(3), 309-336.

Warren, W. H., \& Verbrugge, R. R. (1984). Auditory perception of breaking and bouncing events: a case study in ecological acoustics. Journal of Experimental Psychology: Human perception and performance, 10(5), 704.

Weiskopf, D. A. (2011). Models and mechanisms in psychological explanation. Synthese, 183(3), 313.

Wenderoth, N., Bock, O., \& Krohn, R. (2002). Learning a new bimanual coordination pattern is influenced by existing attractors. Motor Control, 6(2), 166-182.

Wickelgren, E. A., \& Bingham, G. P. (2001). Infant sensitivity to trajectory forms. Journal of Experimental Psychology: Human Perception and Performance, 27(4), 942.

Wilkie, R. M., \& Wann, J. (2003). Controlling steering and judging heading: Retinal flow, visual direction, and extra-retinal information. Journal of Experimental Psychology: Human Perception and Performance, 29, 363-378.

Wimmers, R. H., Beek, P. J., \& van Wieringen, P. C. (1992). Phase transitions in rhythmic tracking movements: A case of unilateral coupling. Human Movement Science, 11(1-2), 217-226.

Wilson, A. D. (2012). The state-of-the-art is healthy; time to take the next step. Developmental Medicine \& Child Neurology, 54(10), 875-876.

Wilson, A. D. (2014). Action scaling of distance perception is task specific and does not predict 'the embodiment of culture': A comment on Soliman, Gibson \& Glenberg (2013). Frontiers in Psychology 5, 302. doi: 10.3389/fpsyg.2014.00302. 
Wilson, A. D. (2018a, July 13). Interface Theory vs Gibson: An Ontological Defence of the Ecological Approach. https://doi.org/10.31234/osf.io/zbkqd

Wilson, A. D. (2018b, November 1). Information is Relational, Affordances Are Not. https://doi.org/10.31234/osf.io/5qb92

Wilson, A. D., \& Bingham, G. P. (2008). Identifying the information for the visual perception of relative phase. Perception \& psychophysics, 70(3), 465-476.

Wilson, A. D., Bingham, G. P., \& Craig, J. C. (2003). Proprioceptive perception of phase variability. Journal of Experimental Psychology: Human Perception and Performance, 29(6), 1179.

Wilson, A. D., Collins, D. R., \& Bingham, G. P. (2005a). Perceptual coupling in rhythmic movement coordination: stable perception leads to stable action. Experimental Brain Research, 164(4), 517-528.

Wilson, A. D., Collins, D. R., \& Bingham, G. P. (2005b). Human movement coordination implicates relative direction as the information for relative phase. Experimental Brain Research, 165(3), 351-361.

Wilson, A. D., Snapp-Childs, W., \& Bingham, G. P. (2010). Perceptual learning immediately yields new stable motor coordination. Journal of Experimental Psychology: Human Perception and Performance, 36(6), 1508.

Wilson, A. D., Zhu, Q., \& Bingham, G. P. (2018) Affordances and the Ecological Approach to Throwing for Long Distances and Accuracy. In The Handbook of Embodied Cognition and Sport Psychology (Cappuccio, Massimiliano (ed.). MIT Press.

Wilson, A. D., \& Golonka, S. (2013). Embodied cognition is not what you think it is. Frontiers in Psychology, 4, 58. 
Wilson, A. D. \& Golonka, S. (2015). Connecting the conceptual dots in embodied cognition: A comment on Dijkstra et al, "How body balance influences political party evaluations: A Wii balance board study". Frontiers in Psychology, 6:853.

Yamanishi, J. I., Kawato, M., \& Suzuki, R. (1979). Studies on human finger tapping neural networks by phase transition curves. Biological cybernetics, 33(4), 199-208.

Yamanishi, J. I., Kawato, M., \& Suzuki, R. (1980). Two coupled oscillators as a model for the coordinated finger tapping by both hands. Biological cybernetics, 37(4), 219-225.

Zaal, F. T., Bingham, G. P., \& Schmidt, R. C. (2000). Visual perception of mean relative phase and phase variability. Journal of Experimental Psychology: Human Perception and Performance, 26(3), 1209.

Zanone, P. G., \& Kelso, J. A. S. (1992). Learning and transfer as dynamical paradigms for behavioral change. In G. E. Stelmach \& J. Requin (Eds.), Advances in psychology, Vol. 87. Tutorials in motor behavior, 2 (pp. 563-582). Oxford, England: North-Holland.

Zanone, P. G., \& Kelso, J. A. S. (1994). The coordination dynamics of learning: Theoretical structure and experimental agenda. In Interlimb Coordination (pp. 461-490).

Zanone, P. G., \& Kelso, J. S. (1997). Coordination dynamics of learning and transfer: collective and component levels. Journal of Experimental Psychology: Human Perception and Performance, 23(5), 1454. 